\title{
Aproximaciones al concepto de Amor y su vínculo con la violencia en los adolescentes: caso de alumnos de bachillerato de la Escuela Superior de Tizayuca
}

\author{
Approaches to concept of Love and its link with violence in adolescents: study case of \\ baccalaureate school students of the Superior School of Tizayuca
}

\author{
Mayra A. Valerio-Nolasco ${ }^{a}$, Luis F. Rivero-Zambrano ${ }^{b}$
}

\begin{abstract}
:
This text aims to frame the concept of Love that students of the high school at Higher Education School Tizayuca have built. It is based on the scoop of that Love concept is founded during the childhood through the experiences lived, and then it became a source of violence during teen-age.
\end{abstract}

Keywords:

Love, violence, teenagers.

\section{Resumen:}

El siguiente escrito pretende enmarcar una aproximación al concepto de amor que los alumnos del programa educativo de bachillerato de la Escuela Superior de Tizayuca UAEH construyen, tomando como primicia que dicho concepto se cimienta a partir de las vivencias que se tienen desde la niñez y como este concepto se convierte en un detonante de violencia en los chicos de edad adolescente.

\section{Palabras Clave:}

Amor, violencia, adolescents.

\section{Introducción}

La presente investigación pretende mostrar un panorama de las problemáticas actuales que se presentan en el contexto "escuela", donde específicamente se investigará a la población adolescente en el nivel bachillerato, entorno a los temas del amor y la violencia.

El tema del amor es confuso e incierto, y aún más en la etapa adolescente donde se está formando una personalidad y carácter de acuerdo a las experiencias que a cada persona le ha tocado vivir.

La violencia es una manifestación de poder, una forma de mostrar la sumisión de las personas por debajo de otras, y esta puede manifestarse de diversas formas, incluso sin saber que esta relación existe.
La Escuela Superior de Tizayuca (ESTi), es una institución de educación media superior y superior, ubicada en la entrada del estado de Hidalgo, siendo este un lugar estratégico debido a que colinda con el Estado de México y muy cerca de la Ciudad de México. El objetivo de esta investigación, es analizar la conceptualización del amor de los alumnos del programa educativo de Bachillerato de la Escuela Superior de Tizayuca, los prejuicios asumidos inconscientemente e identificarlos como tales, y como este concepto impera como detonador de la violencia en dichos sujetos.

Además, se pretende analizar los diversos tipos de violencia vinculadas al amor que se manifiestan en la escuela antes mencionada con la finalidad de emplear estrategias específicas que sean capaces de identificar si son víctimas o victimarios de manera consciente 0 inconsciente a partir del concepto de amor que ellos

Mayra Anaid Valerio Nolasco, Universidad Autónoma del Estado de Hidalgo, Escuela Superior de Tizayuca, Email: mayra_valerio@uaeh.edu.mx

b Luís Francisco Rivero Zambrano, Universidad Autónoma Metropolitana, Escuela Nacional de Biblioteconomía y Archivonomía, Email: 
mismos construyeron, teniendo como referentes su entorno.

\section{El adolescente, el amor y la familia.}

Según la Organización Mundial de la Salud," la adolescencia es el periodo comprendido entre 10 y 19 años, es una etapa compleja de la vida, que marca la transición de la infancia al estado adulto, con ella se producen cambios físicos, psicológicos, biológicos, intelectuales y sociales. Se clasifica en adolescencia, precoz o temprana de 10 a 14 años y la segunda o tardía que comprende entre 15 y 19 años de edad" (Borrás, 2014).

Según lo anterior este intervalo de edad es donde los sujetos sufren una transición de niños a adultos, por ello estos sufren diversos problemas de tipo social como son problemas de socialización al intentar encajar en círculos de amistad, en lo académico donde el sistema de tomar clases cambia de la primaria a la secundaria, teniendo diversidad de profesionales de la educación que les imparten numerosas asignaturas con un mayor nivel de complejidad, en lo emocional, donde sufren cambios bruscos en su personalidad y donde ni ellos mismos comprenden lo que sucede con su sentir, tendiendo un choque de emociones, pero sobre todo de identidad, ya que estos alumnos sufren variedad de cambios que determinarán su personalidad en su vida futura. Se debe señalar que un adolescente no está preparado en experiencia para todo lo que en conjunto le sucede. Por ello es común que presenten diversos problemas en su vida cotidiana.

El concepto de amor se define de acuerdo al constructo de cada persona (Massa, Pat, Keb, Canto, \& Chan, 2011), cada individuo construye su propio concepto de amor, que puede ir desde el deseo carnal, el amor fraternal o el amor maternal, por ello se vuelve a resaltar que el individuo expresa su forma de amar de acuerdo al concepto de amor que le trasmitieron sus familiares, es decir, cómo fue tratado el sujeto durante su niñez, o el aprendizaje que obtuvo del ultimo noviazgo, de la forma en que sus padres demostraban su amor, entre otras cosas.

Bauman en su obra "Amor liquido" menciona otro tipo de amor, el que esta diluido entre la sociedad consumista contemporánea (Bauman, 2005), este tipo de amor según el autor, demuestran la fragilidad de los vínculos humanos y como estas relaciones pasan a segundo término, debido a que solo se requiere cubrir ciertas necesidades y se desecha a la persona, viendo a esta última como un producto y no como persona.
La complejidad del tema del amor implica tratar diversas esferas del comportamiento humano y familiar, donde se vincula el conocimiento previo que tuvo el niño sobre el amor y lo reproduce en la adolescencia; por ello la familia tiene un rol importante en la idea que construyen los adolescentes del amor.

Ahora bien, es preciso mencionar que la violencia siempre ha existido, buena parte de la historia de la humanidad se escribe con sangre humana, donde se utilizó la fuerza para dominar, conquistar y sobrevivir a los enemigos, objetivos que actualmente no tiene la violencia, sino ahora esta se enfoca a otras vertientes como son según (Pérez, González, \& Sánchez, 2015):

$\begin{array}{ll}\text { - } & \text { Violencia para sobrevivir } \\ \text { - } & \text { Violencia para controlar el poder } \\ \text { - } & \text { Violencia para sublevarse contra la dominación } \\ \text { - } & \text { Violencia física } \\ & \text { Violencia física psíquica. }\end{array}$

Cada una de las tipologías de violencia mencionadas, enmarcan un fin distinto, es por ello que se denota que el ser humano tiene una constante lucha contra ser violentado en cualquiera de sus contextos. El amor como ya se mencionó se define de acuerdo al constructo de la concepción de cada persona; la violencia es un elemento integral en la vida de los hombres y mujeres oprimidos de la sociedad, denotando que este binomio está presente las relaciones actuales.

\section{Aproximaciones al concepto del amor como detonador de violencia en alumnos de bachillerato de la ESTi}

"La escuela debería ser un lugar privilegiado para proporcionar una formación que permita participar plenamente en la vida ciudadana y democrática, pero se puede percibir hoy, que existe una contradicción entre el tipo de educación que se proporciona en las escuelas, y el modelo de sociedad al que formalmente se aspira" (Delval, 2013), siendo la escuela un reproductor de las prácticas familiares y sociales aprendidas fuera de esta. Esta reproducción hegemónica en la escuela se basa en tres esferas: desigualdades en relaciones de poder, sexismo y discriminación; de esta forma los estudiantes al llegar a la escuela experimentan un "choque de pensamientos" en donde adoptan el título de dominante o dominado según se den los casos. Según Verdú, la expresión de la desigualdad en lo relacional-afectivo proviene de la reproducción no consciente del sexismo en los guiones amorosos de género (Verdú, 2014), lo anterior, dependiendo de la personalidad y la identidad que se construyó desde el seno familiar. 
Para el desarrollo de este trabajo se realizaron 146 encuestas a estudiantes del programa educativo de bachillerato, de un universo total de 1186 alumnos inscritos, donde la finalidad del instrumento es identificar el concepto de amor de los alumnos y que fue lo que influyó para construir dicho concepto.

Las edades de los encuestados corresponden de los 15 a 18 años, ya que es la edad que oscila en el nivel medio superior, sin embargo, existen alumnos que tiene más de 18 años y aún no han terminado el bachillerato.

\section{¿Qué edad tienes?}

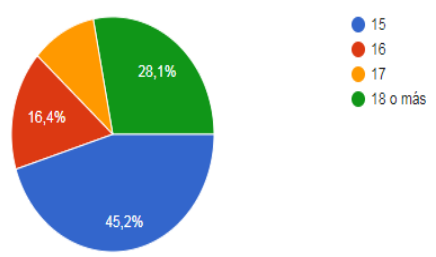

\section{Gráfico 1}

Los resultados nos permiten inferir que el $45.2 \%$ de los alumnos que contestaron a encuesta tiene 15 años (ver gráfico 1), el 28.1\% tienen 18 o más. El 16.4\% tiene 16 años, y solo el $10.3 \%$ tienen 17 años, esto es justificable ya que los alumnos de 15 años están entre primero y segundo semestre y se concentran una mayoría en este rango debido a que fueron aceptados 480 alumnos en primer semestre.

¿Eres hombre o mujer?

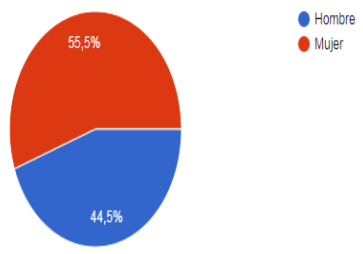

Gráfico 2

Según el gráfico 2 , el $55.5 \%$ de la muestra son mujeres y el $44.5 \%$ son hombres, esto debido a que la encuesta fue contestada por una mayoría en mujeres.

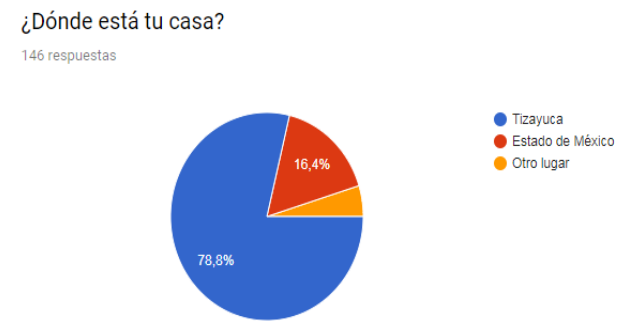

Gráfico 3

Según el gráfico 3, el $78.8 \%$ de los encuestados residen en Tizayuca, el $16.4 \%$ en el Estado de México y solo el $4.8 \%$ comentaron que viven en otro lugar. En los últimos 10 años, Tizayuca ha crecido de manera exponencial donde a medida de este crecimiento, se ha requerido un mayor número de servicios de salud, educación y sobre todo empleo bien remunerado; por ello en la actualidad Tizayuca ha sido catalogado como un municipio "dormitorio" dándole ese nombre debido a que la mayoría de los adultos trabajan todo el día y solo llegan a dormir y los hijos permanecen en casa solos o en compañía de otros familiares, esta es una constante en fraccionamientos como Haciendas de Tizayuca, Rancho Don Antonio, entre otros. Siendo esta problemática de suma importancia para el objeto que se está estudiando.

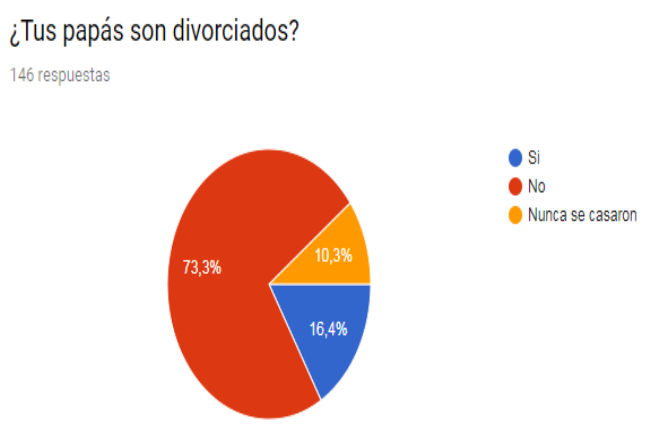

Gráfico 4

El grafico 4, el $73.3 \%$ de los encuestados manifestaron que sus papas están casados, el $16.4 \%$ están divorciados y el $10.3 \%$ comentan nunca se casaron. Esta cuestión se realizó con la intensión de que se observará "la imagen" que los encuestados tienen sobre el amor en su seno familiar, observándose que el $73.3 \%$ de ellos tienen el concepto familiar de: papá, mamá e hijos. 
¿Vives algún tipo de violencia dentro de tu casa? (Física, psicológica, simbólica, patrimonial, sexual.)

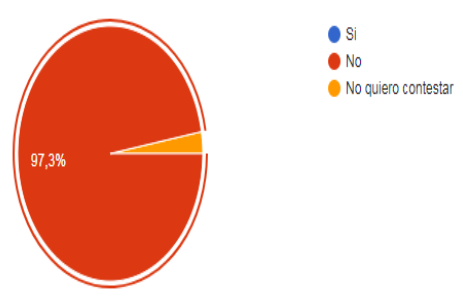

Gráfico 5

Es posible ver en el gráfico que el $97.3 \%$ de los encuestados demostraron que no viven algún tipo de violencia en casa y el $2.7 \%$ restante no quiso contestar, este último porcentaje deja la posibilidad de que los adolescentes si están viviendo algún tipo de violencia en su casa. El amor no debería ser problema, presión, sufrimiento y malos tratos, los adolescentes están en una etapa vulnerable en donde: la familia, los "amigos", las parejas sentimentales, las redes sociales, la moda, la música, entre muchas cosas más influyen en su personalidad, y sus decisiones de vida y el concepto que tienen de la realidad.

\section{¿Cuándo tienes dudas relacionadas al amor con quien lo consultas?} 146 respuestas

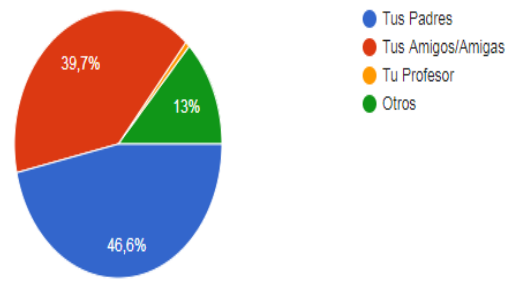

\section{Gráfico 6}

El $46.6 \%$ de los encuestados consultan sus dudas relacionadas al amor con sus padres, el $39.7 \%$ con sus amigos, el $13 \%$ con otros y el $0.7 \%$ restante con sus profesores. Se puede observar que el $39.7 \%$ de los sujetos acuden a sus profesores para platicar sobre sus inquietudes relacionadas con el amor, siendo esto un detonante en el concepto que ellos construyen sobre el amor. Es muy claro que casi el $40 \%$ de los encuestados construyen sus referentes relacionados al amor con sus amigos (ver gráfico 6).

\section{¿Te da pena consultar platicar con tus papas sobre el amor? \\ 144 respuestas}

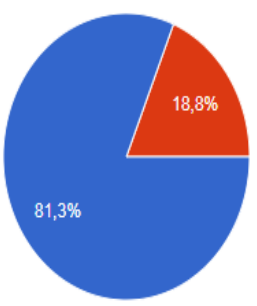

no

Gráfico 7

El $81.1 \%$ de los encuestados no les apena platicar sobre el amor con sus padres, y el $18.8 \%$ restantes si les apena (ver gráfico 7). Este dato es relevante debido a que las personas con quien conversen sobre amor, influirán sobre su conceptualización del amor.

\section{¿Qué tipo de programas comúnmente ves?} 146 respuestas
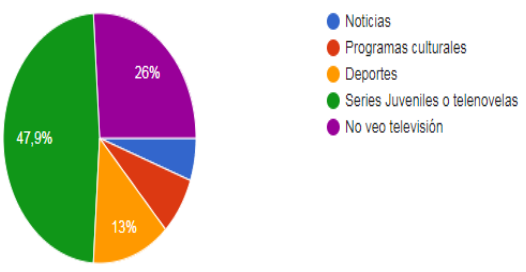

Gráfico 8

El $47.9 \%$ de los encuestados ven series juveniles o telenovelas, el $26 \%$ no ven televisión, el $13 \%$ ven deportes, el $7.5 \%$ ven programas culturales y el $5.5 \%$ restante ven noticias, siendo detonante este dato, ya que se observa que los medios de comunicación y programas juveniles (ver gráfico 8). El impacto que tienen los medios de comunicación en la concepción del amor produce el enlace de las emociones románticas con la cultura, la economía y la organización social del capitalismo avanzado: "Eva Illouz afirma que dicha intersección se da mediante dos procesos: la romantización de los bienes de consumo y la mercantilización del romance. Esto es, el papel del romance en la construcción de mercados de consumo masivo y los modos en los que se produce la incorporación de las prácticas económicas a las prácticas amorosas" (Panadero, 2016) 
¿Consideras que es normal que las parejas prohíban tener amigos y amigas?

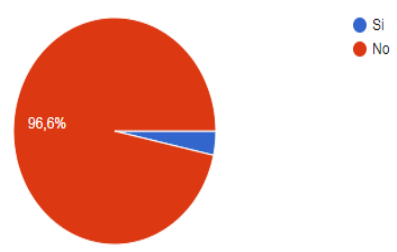

Grafico 9

El grafico 9, nos permite observar que el $96.6 \%$ de los encuestados no consideran que es normal que las parejas prohíban tener amigos y amigas, el otro $3.4 \% \mathrm{Si}$ lo consideran, demostrando con este último dato que, los sujetos tienen un concepto de amor relacionado con las prohibiciones y el control, expresando una dominación sobre el sujeto. Respecto al tema de la dominación, Bourdieu plantea que "se legitima una relación de dominación en una naturaleza biológica que es sí misma una construcción social naturalizada" (Bourdieu, 1999) y aunque Bourdieu específicamente se refiere a la dominación masculina, se puede validar esta afirmación debido a la sumisión del sujeto, ya sea hombre o mujer.

\section{Conclusiones}

El estudio realizado permite identificar:

1) El concepto de amor de los alumnos de Bachillerato de la Escuela Superior de Tizayuca.

2) Los tipos de violencia que se ejerce a partir del concepto de amor

3) Identificar si los alumnos están sometidos a un sistema de dominación a partir del concepto que construyeron de amor

1) El concepto de amor de los alumnos de Bachillerato de la Escuela Superior de Tizayuca, donde se encontró que los alumnos construyeron su concepto de amor a partir de tres predominios: La familia, los amigos y los medios de comunicación. Se observó que el $73.3 \%$ de los alumnos encuestados tienen el modelo de una familiatradicional: papá, mamá e hijos; el $39.7 \%$ de los encuestados hablan de amor con sus amistades, eso indica que parte de su concepto de amor se ve influenciado por la asimilación de otras personas y el $47.9 \%$ de los encuestados, comentaron que veían telenovelas y series juveniles, denotando que la asimilación del amor "telenovelesco" se convierte en parte fundamental de su conceptualización, entendiendo como "telenovelesco" aquel amor que generalmente se observa en televisión: amores que se desarrollan envueltos en situaciones problemáticas, engañosas o violentas, pero que tienen en un final feliz.

2) Los tipos de violencia que se ejercen a partir del concepto de amor se pueden definir desde diferentes perspectivas, en un primer momento se propone el concepto de amor violento para describir la privatización de las libertades del individuo para ejercer una dominación, este genera en los estudiantes un ambiente hostil, desmotivación académica y conflictos en relaciones interpersonales; esta problemática no solo se refleja en la parte académica, sino que las luchas de poderes, gritos, bullying, entre otras agresiones, son parte de la vivencia y donde ya ni siquiera los alumnos, profesores y autoridades se percatan de que existe este "amor violento" por la necesidad de sentirse aceptados, y de aquí se parte el siguiente concepto Bourdiano que refiere a la violencia simbólica donde se refiere a esa dominación que el dominante otorga al dominador sin pensarlo y se presenta en un estado de habitus que ni siquiera se observa la relación de dominación.

3) La conceptualización de amor que se puede construir a través del instrumento implementado es diversa, las interpretaciones fueron:

$\checkmark$ Sentimiento por alguna persona

$\checkmark$ Cariño, preocupaciones, felicidad

$\checkmark$ Es llorar cuando nos dice adiós

$\checkmark$ Amor es pensar en el bien del otro, sin compromiso

$\checkmark$ El amor es disfrutar cada momento con al gente que te rodean

$\checkmark \quad$ Yo no nací para amar.

Con lo anterior se puede observar que los alumnos ya tienen marcado un concepto del amor construido, que tiene que ver con el amor para la pareja, aunque se debe recalcar que en ningún momento se especificó que era amor de pareja, ningún encuestado se refirió sobre el amor a sus padres o amigos.

Se debe comentar que este estudio queda abierto a un análisis más amplio, solo está limitado a la aproximación de la construcción del concepto de amor y queda abierto al análisis sobre el amor en la "Sociedad Red" (Castell 2001 cit. por (Blanco, 2014) donde ya se permite la comunicación a través de redes sociales y donde en poco tiempo estos medios de comunicación ha rebasado sus expectativas de usuarios, ya que cualquier persona de cualquier edad y medio económico puede acceder a ella y precisamente por estos medios es donde surge otra nueva forma de violencia, específicamente en el nivel 
secundaria y medio superior, que van desde ofender a un alumno en las redes sociales, hacer memes, publicar packs o simplemente desprestigiar a sus compañeros.

\section{Referencias}

Aguilar, C. L. (20 de Diciembre de 2010). Inmujeres.gob. Recuperado el 10 de Junio de 2017, de Manual para la prevención de la violencia. Amor y Violencia en el Noviazgo: http://cedoc.inmujeres.gob.mx/ftpg/Tabasco/tabmeta13_6.pdf

Bauman, Z. (2005). Amor líquido. Acerca de la fragilidad de los vínculos humanos. Argentina: Fondo de Cultura Económica.

Blanco, R. M. (2014). Instituto de la Comunicación e Imagen. Universidad de Chile. Recuperado el 17 de 06 de 2017, de Implicaciones del Uso de las Redes Sociales en el Aumento de la Violencian de Género: http://revistas.uchile.cl/index.php/RCM/article/viewFile/32375/37657

Borrás, T. (2014). SCIELO. Recuperado el 17 de 06 de 2017, de Adolescencia: Definición, vulnerabilidad y oportunidad: http://scielo.sld.cu/pdf/ccm/v18n1/ccm02114.pdf

Bourdiau, P (199). La Dominción Masculina. Francia: Editorial Barcelona.

Castillo , A., \& Valles, R. (2015). Revista Científica de la Universidad Autónoma de Manizales. Recuperado el 2017 de 06 de 2017, de Comprensión y Socialización del concepto de género en estudiantes de educación media superior en Pachuca, México: http://publicaciones.autonoma.edu.co/index.php/anfora/article/view/19 $/ 17$

Castro, R. B. (10 de Octubre de 2008). El amor como concepto filosófico y práctica de vida, entrevista con Edgar Morales. Recuperado el 10 de Junio de 2017, de Revista Digital Universitaria UNAM http://www.revista.unam.mx/vol.9/num11/art92/art92.pdf

Delval, J. (2013). La Escuela para el siglo XX1. SCielo, 1-10.

Díaz-Aguado, J. M. (2005). La violencia entre Iguales en la adolescencia y su prevencón desde la escuela. Recuperado el 2017 de Junio de 10, de
Revista
Psicothema:

https://www.unioviedo.es/reunido/index.php/PST/article/view/8310

Fernández, I. (2013). Amor, sexualidad y adolescencia. Recuperado el 17 de 06 de 2017, de Dialnet: https://dialnet.unirioja.es/descarga/articulo/5144548.pd

Ferrer, P. V., \& Bosch, E. (Abril de 2013). Profesorado. Revista de Curriculum y Formación del Profesorado. Recuperado el 17 de 06 de 2017, de Del Amor Romantico a la Violencia de Género. Para una coeducación emocional en la agenda educativa: https://recyt.fecyt.es/index.php/profesorado/article/view/41570/23660

Galicia, I., Sánchez, A., \& Robles, F. (Mayo de 2013). Psicología desde el Caribe; Barranquilla. Recuperado el 17 de 06 de 2017, de Relaciones entre estilos e amor y violencia en adolescentes: http://search.proquest.com/openview/41d0bd846c533973e461e525109 022af $/ 1$ ?pq-origsite $=$ gscholar $\&$ cbl $=2027439$

González, C. M. (2007). Violencia en el Noviazgo: Un Estudio Exploratorio entre estudiantes universitarias (1 ed.). México, Nuevo León: UANL.

Massa, J., Pat, Y. M., Keb, R. A., Canto, M. V., \& Chan, N. (Septiembre de 2011). La definición de Amor y Depednecia Emocional en Adolescentes de Mérida, Yucatán. Recuperado el 2017 de 06 de 2017, de Revista
Eléctronica de Psicología Iztacala: http://www.medigraphic.com/pdfs/epsicologia/epi-2011/epi113k.pdf

Panadero, E. (2016). La utopía romántica y las contradicciones. España: Universidad a Navarra.

Pérez, V. M., González, A. G., \& Sánchez, C. V. (Marzo de 2015). $3^{\circ}$ Congreso Virtual Internacional sobre Cuerpos Académicos y Grupos de Investigación. Recuperado el 27 de Junio de 2017, de Las emociones que son detonadas por la violencia: http://pag.org.mx/index.php/PAG/article/view/475

Ramos, C., Rodríguez, M. d., Gonzalbo, P., Giraud, F., Alberro, S., Carner, F., y otros. (2006). Presecia y transparencia: La mujer en la Historia de México. México: El Colegio de México.

Rodríguez, Y., Lameiras, M., \& Carrera, M. (2015). Revista de Estudios eInvestigación en Psicologia y Educación. Recuperado el 17 de 06 de 2017, de Amor y Sexismo: Una peligrosa relación en los y las adolescentes gallegos/as: http://revistas.udc.es/index.php/reipe/article/view/234/pdf_13

Saltijeral, M. T., Ramos, L., \& Caballero, M. (Abril de 1998). Las Mujeres que han sido victimas de maltrato conyugal: tipos de violencia experimentada y algunos efectos de la salud mental. Recuperado el Junio de 2017, de Salud Mental volumen 21: http://www.inprfcd.gob.mx/pdf/sm2102/sm210210.pdf

Varela, V. G. (2004). Revista Uruguaya de Psicoanálisis. Recuperado el 17 de 06 de 2017, de El amor enla adolescencia (Los adolescentes que no pueden amar): http://www.apuruguay.org/revista_pdf/rup99/rup99varela.pdf

Verdú, D. A. (Marzo de 2014). Igualdad y Desigualdad de Género en los imaginarios Sociales del amor de Pareja Heterosexual. Recuperado el 17 de 06 de 2017, de ULE Revistas Universidad de León: http://revpubli.unileon.es/ojs/index.php/cuestionesdegenero/article/vie w/1014/1034

Zurbano , B., Lineria, I., \& Campos, B. (2015). Oñati Socio-Legal. Recuperado el 17 de 06 de 2017, de Concepto y Representación de la Violencia de Género: Reflexiones sobre el Impacto de la población Joven: https://idus.us.es/xmlui/bitstream/handle/11441/46369/SSRNid2612467.pdf?sequence $=1$ \&isAllowed $=y$ 